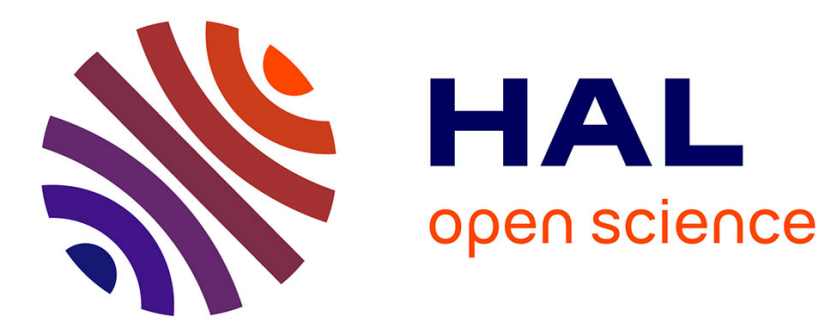

\title{
Conseiller un patient souffrant d'hypersensibilité dentinaire
}

\author{
Jacques Buxeraud
}

\section{To cite this version:}

Jacques Buxeraud. Conseiller un patient souffrant d'hypersensibilité dentinaire. Actualités Pharmaceutiques, 2019, 58, pp.49 - 51. 10.1016/j.actpha.2019.02.010 . hal-03484652

\section{HAL Id: hal-03484652 \\ https://hal.science/hal-03484652}

Submitted on 21 Dec 2021

HAL is a multi-disciplinary open access archive for the deposit and dissemination of scientific research documents, whether they are published or not. The documents may come from teaching and research institutions in France or abroad, or from public or private research centers.
L'archive ouverte pluridisciplinaire HAL, est destinée au dépôt et à la diffusion de documents scientifiques de niveau recherche, publiés ou non, émanant des établissements d'enseignement et de recherche français ou étrangers, des laboratoires publics ou privés.

\section{다)(1) $(5$}

Distributed under a Creative Commons Attribution - NonCommerciall 4.0 International 
Dochead pratique

Sous-dochead bucco-dentaire

\section{Conseiller un patient se plaignant de vives douleurs dentaires au chaud et au froid}

\section{Jacques Buxeraud}

Professeur émérite des Universités

Faculté pharmacie de Limoges, 2 rue du Docteur-Marcland, 87025 Limoges cedex, France

Adresse e-mail : jacques.buxeraud@unilim.fr (J. Buxeraud).

\section{Résumé}

Un patient qui ressent de vives douleurs dentaires au chaud et au froid souffre le plus souvent d'hypersensibilité dentinaire. Le pharmacien doit être capable de détecter cette affection afin de lui conseiller les soins appropriés et de l’orienter, si nécessaire, vers un chirurgien-dentiste.

(C) 2019

Mots clés - conseil officinal ; dentifrice désensibilisant ; hypersensibilité dentinaire

\section{Summary à venir}

(C) 2019

Keywords à venir

L'hypersensibilité dentinaire se définit comme une douleur brève et vive résultant de l'exposition de la dentine en réponse à des stimuli, généralement thermiques, évaporatifs, tactiles, osmotiques ou chimiques, et qui ne peut être attribuée à aucun autre défaut ou maladie dentaire. Une carie dentaire (douleur pulsatile plus ou moins continue) ou une suite de chocs dentaires (traumatismes pouvant occasionner une fissure) nécessitent une consultation pour s'assurer du diagnostic différentiel. II importe donc de toujours vérifier la date de la dernière consultation dentaire avant de dispenser un conseil.

\section{T1 La dentine}

TEG1 La dentine est un tissu calcifié, jaunâtre et dur de la dent, qui se situe sous l'émail au niveau de la couronne, partie visible de la dent, et sous le cément au niveau de la racine dans la gencive (figure 1). 
TEG1 Sa structure est poreuse, traversée par des canaux microscopiques appelés tubules ou canalicules, qui transmettent des informations aux nerfs sous-jacents, par exemple en cas de choc thermique (froid ou chaud).

TEG1 Selon la théorie hydrodynamique, la sensation de douleur serait due à des changements de débit et/ou de volume du fluide dans les canalicules dentinaires (encadré 1), conséquence de l'activation de mécanorécepteurs dans les nerfs intratubulaires ou dans la pulpe superficielle.

\section{Encadré 1. Les canalicules dentinaires}

Les canalicules dentinaires sont des petits canaux microscopiques perpendiculaires au canal principal de la dent. Ils ont une extrémité rejoignant la pulpe, l'autre située en surface de dentine.

Sur deux colonnes près de son appel (ou 1 colonne + marge)

\section{T1 L’hypersensibilité dentinaire, une affection fréquente}

TEG1 Touchant un très grand nombre de patients, puisqu' elle concernerait 10 à $30 \%$ des adultes, l'hypersensibilité dentinaire provoque une douleur aiguë de très courte durée en réponse à divers stimuli. Elle peut se manifester à tout âge, mais la majorité des patients ont entre 20 et 50 ans, un pic prévalence étant observé chez les 30-39 ans.

TEG1 Cette affection peut affecter la qualité de vie au quotidien : la consommation de glaces et de boissons, ainsi qu'un simple brossage des dents peuvent s'avérer douloureux.

TEG1 Les canines et les premières prémolaires sont les plus fréquemment touchées, suivies des incisives et des deuxièmes prémolaires. Les molaires sont souvent épargnées.

TEG1 Les régions cervicales des dents sont préférentiellement impactées. Toutefois, les sites occlusaux le sont de plus en plus chez les jeunes adultes.

\section{T1 L'origine de la douleur}

TEG1 La très vive douleur ressentie par le patient au niveau des dents est due à la mise à nu de la dentine au niveau des collets, qui laisse apparaître les canalicules dentinaires qui se retrouvent ainsi directement au contact de la cavité buccale (encadré 2).

TEG1 Les causes de ce phénomène sont :

- soit la perte de la couche d'émail protectrice, laissant apparaître la dentine ;

- soit la mise à nu des collets, qui entraîne une exposition du cément recouvrant la partie radiculaire de la dent alors rapidement éliminée par l'abrasion provoquée par le brossage et laisse apparaître la dentine sous-jacente.

TEG1 La douleur engendrée lors du brossage dissuade rapidement les personnes de se brosser les dents : la plaque dentaire peut alors s'accumuler et les caries se développer d'autant plus vite que la 
dentine constitue un tissu moins minéralisé, donc moins résistant que l'émail. Il est question de caries dentinaires ou de caries cervicales quand elles se situent au niveau du collet.

TEG1 L'accumulation de plaque favorise également l'inflammation gingivale qui engendre une récession gingivale et augmente ainsi l'hypersensibilité : c'est le cercle vicieux de l'hypersensibilité.

\section{Encadré 2. Facteurs associés à l’hypersensibilité dentinaire}

- Récession gingivale

- Maladie parodontale

- Fissures profondes des dents

- Perte d'émail, de cément et de dentine pouvant être dues à l'érosion chimique, à l'abrasion mécanique ou même à une fracture de la dent

Sur deux colonnes près de son appel (ou 1 colonne + marge)

\section{T1 Les signes cliniques de l'hypersensibilité dentinaire}

TEG1 Plusieurs signes sont évocateurs d'une hypersensibilité dentinaire, notamment une sensation douloureuse ressentie :

- au niveau des dents, d'apparition et de disparition rapides ;

- lors du seul contact d'une crème glacée contre les dents ou d'une respiration buccale en hiver ;

- lors de la prise d'un produit acide.

- au contact d'une fourchette ou d'une brosse à dents.

TEG1 La sévérité de la douleur va de la simple sensation désagréable à la douleur sévère pouvant altérer le quotidien des patients. Les épisodes algiques sont d'intensité et de fréquence variables et ne peuvent pas être expliqués par d'autres pathologies (encadré 3).

Encadré 3. Les bonnes questions à poser au patient à l'officine pour détecter une hypersensibilité dentinaire

- Depuis combien de temps ressentez-vous ces douleurs?

- Avez-vous remarqué si elles surviennent à certains moments précis (contact avec le froid, le chaud, l'air, lors du brossage des dents...) ?

- Comment pouvez-vous décrire la douleur que vous ressentez (violente, brève...) ?

- Avez-vous des problèmes de gencives (saignements, déchaussement) ?

- Quand avez-vous réalisé votre dernier contrôle dentaire chez votre dentiste ? 
- Quel type de brosse à dents utilisez-vous ?

- Comment et à quelle fréquence vous brossez-vous les dents?

- Quel dentifrice utilisez-vous ?

- Consultez-vous régulièrement votre chirurgien-dentiste?

Sur deux colonnes près de son appel (ou 1 colonne + marge)

\section{T1 Prendre en charge l'hypersensibilité dentinaire}

TEG1 La gestion de l'hypersensibilité dentinaire comprend plusieurs étapes :

- un diagnostic correct, compatible avec la description clinique de l'hypersensibilité dentinaire, basé sur les antécédents du patient et un examen dentaire ;

- un diagnostic différentiel, afin de s'assurer que les autres conditions pouvant engendrer une douleur similaire sont exclues;

- le traitement approprié de toute condition secondaire qui pourrait induire des symptômes similaires à ceux d'une hypersensibilité dentinaire ;

- l'identification, ainsi que l'élimination ou la minimisation des facteurs étiologiques et prédisposants (habitudes alimentaires, hygiène buccale...) ;

- la recommandation d'un traitement en fonction des besoins individuels [1].

TEG1 En première intention, il convient de recommander des produits d'hygiène buccale spécifiques "dents sensibles" en rappelant les techniques de brossage non iatrogènes (pas de brossage "horizontal"). Deux types de mécanismes d'action entrent en jeu :

- obturant : fluor, notamment d'amines et d'étain, arginine/carbonate de calcium qui obturent les canalicules dentinaires en formant une couche protectrice à la surface de la dentine ;

- désensibilisant : potassium qui réduit le potentiel d'excitation des nerfs pulpaires.

Une solution dentaire "dents sensibles" en traitement d'attaque couplée au dentifrice apporte des résultats en cas d'hypersensibilité sévère. Il est également primordial d'utiliser une brosse à dents douce pour éviter tout phénomène abrasif ou déclencheur.

L'arginine, présente naturellement dans la salive [2] est l'un des vingt acides aminés. Elle est classée comme un acide aminé semi-essentiel. Lorsqu'elle est correctement formulée dans des produits de soins bucco-dentaires (dentifrices et solutions), l'arginine associée au carbonate de calcium est capable d'obturer et de sceller les canalicules dentinaires ouverts, apportant ainsi un soulagement à I'hypersensibilité de la dentine (figure 2). Par attraction électrostatique de l'arginine vers la dentine, I'application de cette association provoque la formation rapide d'un précipité naturel de phosphate et calcium à la surface de la dentine, obturant massivement les canalicules dentinaires. L'apaisement est immédiat (60 secondes) et durable (plusieurs semaines) [3,4]. L'utilisation de produits basés sur 
cette technologie procure un soulagement supérieur de l'hypersensibilité de la dentine par rapport à celle de produits ne comportant pas d'agent désensibilisant ou contenant d'autres agents de désensibilisation (potassium, autres agents obturants).

\section{TEG1 Le chirurgien-dentiste peut être amené à utiliser des techniques d'obturation au cabinet} dentaire en cas d'échec du traitement de première intention. L'obturation des canalicules empêche, en effet, les mouvements des fluides dentinaires et, par conséquent, l'induction de l'influx nerveux à I'origine de la douleur.

\section{T1 Conclusion}

Le patient atteint d'hypersensibilité dentinaire doit comprendre le rôle essentiel joué par l'hygiène buccale quotidienne, ainsi que la nécessité de réduire les facteurs de risque parodontaux en maintenant un bon contrôle de maladies systémiques (diabète) et d'arrêter de fumer (figure 3)

\section{Points à retenir}

- L'hypersensibilité dentinaire se manifeste par une douleur brève et vive résultant de l'exposition de la dentine en réponse à des stimuli (chaud, froid...).

- La dentine est un tissu calcifié et dur de la dent dont la structure, poreuse, est traversée par des canaux microscopiques qui transmettent des informations aux nerfs sous-jacents, par exemple en cas de choc thermique.

- L'hypersensibilité dentinaire peut se manifester à tout âge, mais la majorité des patients ont de 20 à 50 ans.

- Le chirurgien-dentiste peut obturer les canalicules dentinaires.

- Lorsqu'elle est correctement formulée dans des produits de soins bucco-dentaires, l'arginine associée au carbonate de calcium est capable d'obturer et de sceller les canalicules dentinaires ouverts, apportant ainsi un soulagement à l'hypersensibilité de la dentine.

Sur deux colonnes à droite après la puce de fin

\section{Références}

[1] Gillam D, Chesters R, Attrill D et al. Dentine hypersensitivity - guidelines for the management of a common oral health problem. Dent Update. 2013;40(7):523-4.

[2] Panagakos FS, Delgado E, Morrison BM et al. Advanced dentin hypersensitivity technology: a comprehensive review of the benefits of Pro-Argin ${ }^{\text {TM }}$ oral care products. J Clin Dent.

2016;27(Suppl):S1-15. 
[3] Nathoo S, Delgado E, Zhang YP et al. Comparing the efficacy in providing instant relief of dentin hypersensitivity of a new toothpaste containing $8.0 \%$ arginine, calcium carbonate and $1450 \mathrm{ppm}$ fluoride relative to a sensitive toothpaste containing $2 \%$ potassium ion and $1450 \mathrm{ppm}$ fluoride, and a control toothpaste with 1450 ppm fluoride: A threeday clinical study in New Jersey, USA. J Clin Dent. 2009;20(Spec Iss):123-30.

[4] Docimo R, Montesani L, Maturo P et al. Comparing the efficacy in reducing dentin hypersensitivity of a new toothpaste containing $8.0 \%$ arginine, calcium carbonate, and $1450 \mathrm{ppm}$ fluoride to a commercial sensitive toothpaste containing $2 \%$ potassium ion: An eight-week clinical study in Rome, Italy. J Clin Dent. 2009; 20 (Spec Iss):17-22.

Déclaration de liens d'intérêts

L'auteur déclare ne pas avoir de liens d'intérêts.

\section{Figures}

\section{Bux_fig1}

Figure 1. Schéma de la dent avec sa dentine.

(C) Gaba

Sur deux colonnes près de son appel

\section{Bux_fig2}

Figure 2. La dentine avant et après traitement par agents obturants.

(c) Gaba

Sur deux colonnes près de son appel

\section{Bux_fig3}

Figure 3. Conseils d'hygiène bucco-dentaire en cas d'hypersensibilité dentinaire.

(C) J. Buxeraud/Elsevier Masson SAS

Sur trois colonnes près de son appel 
Émail

Dentine

Nerfs

Dentine exposée Canalicules dentinaires ouverts
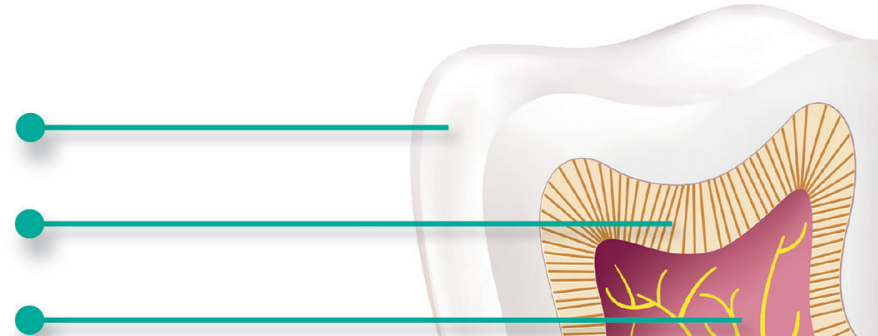


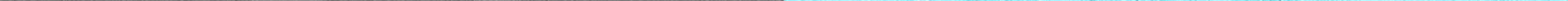




\section{Conseils}

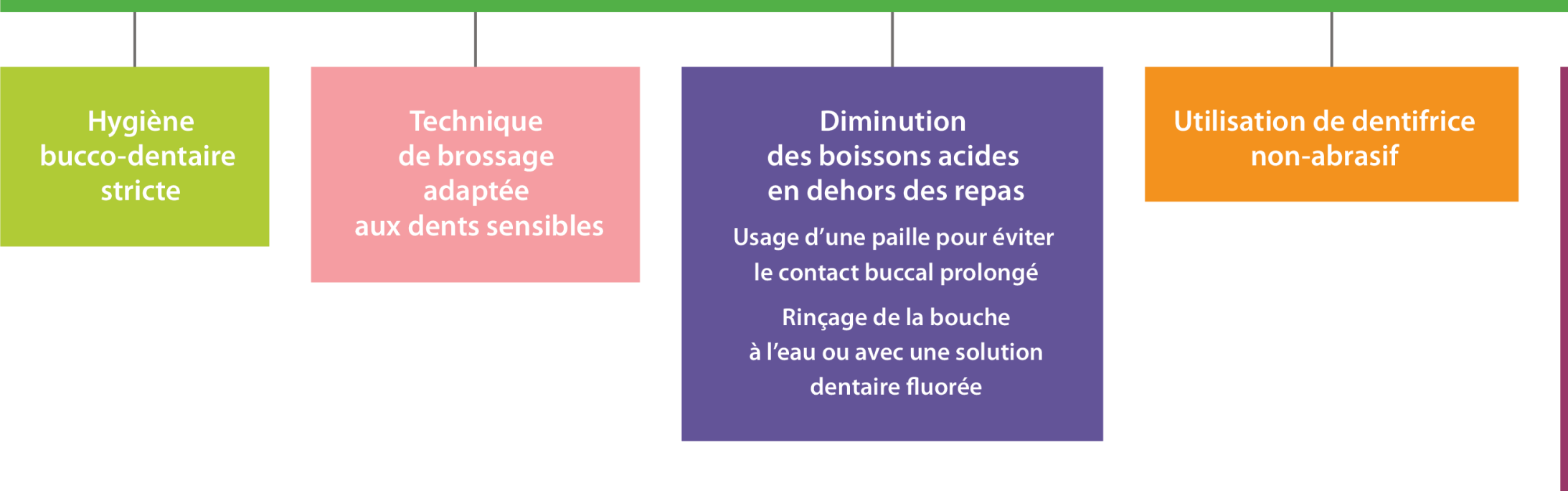

Utilisation de dentifrice

désensibilisant à base de :

- sels de potassium

- sels de strontium

- fluorures d'amine ou d'étain

- arginine/carbonate de calcium

Brossage deux fois par jour

pendant au moins deux semaines

pour apporter un soulagement

significatif

Consultation régulière chez un chirurgien dentiste 